\title{
ASO Author Reflections: ASCL2, a Marker of Dynamic Colon Stem Cell, Involved in Wnt Pathway Activation Reflects Anti- cancer Drug Resistance in Primary Colorectal Cancer
}

\author{
Toshimichi Tanaka, $\mathrm{MD}^{1}$, and Keishi Yamashita, $\mathrm{MD}, \mathbf{P h D}^{2,3}$ \\ ${ }^{1}$ Department of Surgery, Kitasato University School of Medicine, Sagamihara, Kanagawa, Japan; ${ }^{2}$ Epigenetic Treatment \\ Research Group, Chiyoda-ku, Tokyo, Japan; ${ }^{3}$ Division of Advanced Surgical Oncology, Department of Research and \\ Development Center for New Medical Frontiers, Kitasato University School of Medicine, Sagamihara, Kanagawa, Japan
}

\section{PAST}

A portion of colorectal cancer (CRC) is resistant to anticancer treatment. The molecular mechanisms involved in drug resistance must be clarified to develop new therapeutic strategies and biomarkers.

\section{PRESENT}

We applied a phenylbutyrate administration model, previously used for exploration of genes involved in drug resistance in breast cancer and searched novel genes explaining drug resistance to CRC. ${ }^{1}$ In the current study, ASCL2 was significantly associated with resistance against phenylbutyrate, 5-FU, and radiotherapy. ${ }^{2}$ The regulation of ASCL2 may provide a clue for the molecular understanding of CRC treatment failure.

\section{FUTURE}

Ascl2 is a marker of dynamic stem cell of goblet cells of the colorectal noncancerous mucosa. Recently, ASCL2 expression was mainly regulated by long noncoding RNA of WiNTRLINC1 in CRC, which is a direct target of Wnt pathways. $^{3}$ Establishment of control of Wnt pathway, including WiNTRLNC1-ASCL2 axis, may greatly contribute to suppression of CRC from therapeutic point of view through understanding genetic linkage of normal stem cells to cancer occurrence in the future.

DISCLOSURES Toshimichi Tanaka and Keishi Yamashita have no conflicts of interest to disclose.

\section{REFERENCES}

1. Kikuchi M, Yamashita K, Waraya M, et al. Epigenetic regulation of ZEB1-RAB25/ESRP1 axis plays a critical role in phenylbutyrate treatment-resistant breast cancer. Oncotarget. 2016;7:1741-53.

2. Tanaka T, Kojima K, Yokota K, et al. Comprehensive genetic search to clarify the molecular mechanism of drug resistance identifies ASCL2-LEF1/TSPAN8 axis in colorectal cancer. Ann Surg Oncol. 2019;26:1401-11.

3. Giakountis A, Moulos P, Zarkou V, et al. A positive regulatory loop between a Wnt-regulated non-coding RNA and ASCL2 controls intestinal stem cell fate. Cell Rep. 2016;15:2588-96.

Publisher's Note Springer Nature remains neutral with regard to jurisdictional claims in published maps and institutional affiliations.

ASO Author Reflections is a brief invited commentary on the article, "Comprehensive Genetic Search to Clarify the Molecular Mechanism of Drug Resistance Identifies ASCL2-LEF1/TSPAN8 Axis in Colorectal Cancer," Ann Surg Oncol. 2019; 26: 1401-11.

(C) Society of Surgical Oncology 2019

First Received: 13 May 2019;

Published Online: 13 June 2019

K. Yamashita, MD, PhD

e-mail: keishi23@med.kitasato-u.ac.jp 